\title{
Heat and Mass Transfer on the Unsteady MHD Flow of Chemically Reacting Micropolar Fluid with Radiation and Joule Heating
}

\author{
Shamshuddin MD. \\ Department of Mathematics, Vaagdevi College of Engineering, Warangal, India \\ Email address: \\ shammaths@gmail.com \\ To cite this article: \\ Shamshuddin MD. Heat and Mass Transfer on the Unsteady MHD Flow of Chemically Reacting Micropolar Fluid with Radiation and Joule \\ Heating. International Journal of Theoretical and Applied Mathematics. Vol. 3, No. 3, 2017, pp. 110-121. doi: 10.11648/j.ijtam.20170303.13
}

Received: March 14, 2017; Accepted: April 14, 2017; Published: May 22, 2017

\begin{abstract}
To explore numerical simulation of transport in rheological materials processing, in the current paper, a finite element computational solution is presented for magnetohydrodynamic (MHD), incompressible, radiative and chemicallyreacting micropolar fluid flow, heat and mass transfer adjacent to a vertical porous plate embedded in a saturated homogenous porous medium. Rosseland's diffusion approximation is used to describe the radiative heat flux in the energy equation. A Darcy model is employed for the porous medium. The homogeneous chemical reaction of first order is accounted for in the mass diffusion equation. The numerical solutions of the system of non-linear partial differential equations which are rendered into non-dimensional form are obtained using a Galerkin formulation with a weighted residual scheme. The impact of Eringen coupling number, radiation-conduction number, chemical reaction parameter, plate moving velocity parameter, magnetic parameter, thermal Grashof number, species (solutal) Grashof number, permeability parameter, Eckert number on linear velocity, micro-rotation, temperature and concentration profiles. Furthermore, the influence of selected thermo-physical parameters on friction factor, surface heat transfer and mass transfer rate is also tabulated. The finite element solutions are verified with solutions from several limiting cases in the literature. Interesting features in the flow are identified and interpreted.
\end{abstract}

Keywords: Radiative Heat Transfer, Chemical Reaction, Joule Dissipation, Buoyancy, Micropolar Fluid, FEM

\section{Introduction}

The flow of non-Newtonian fluid feature widely in an extensive range of technological applications including, food processing, plastic fabrication, biotechnology and paint emulsion manufacture. To simulate the complex shear stressstrain characteristics of such fluids, numerous mathematical models have been developed. Researchers in this area were initiated by Eringen [1] introduced the microfluid model and later simplified this model to micropolar fluids which can describe sophisticated phenomena including couple stresses, body couples and exhibit gyratory motions, which cannot be analyzed with simpler non-Newtonian models and continued up to now in various case studies. Many recent aspects of micropolar hydrodynamics are documented in Eringen [2] and Lukaswiascz [3]. Extensive discussion of other applications in chemical and mechanical engineering are available in the articles of Airman et al. [4, 5].
The current study is relevant to high temperature electromagnetic rheological flows in energy generators and magneto-rheological materials fabrication systems (where thermal radiation heat transfer is also significant). It is known that they arise in many diverse areas of technology including combustion in gas turbines, convective flows setup where the bounding surfaces absorb heat by solar radiation, design of efficient heat exchangers, etc. These flows require a more sophisticated approach to radiative heat transfer in the system which can substantially influence performance and modify characteristics of manufactured products. In addition to this, such regimes are strongly influenced by thermal boundary conditions. Earlier Raptis and Perdikis [6] presented the presence of radiation in viscoelastic flow. An important analysis in this regard was presented by Abo-Eldahab and Ghonaim [7] who addressed thermal radiation effects in heat transfer of a micropolar fluid through a porous medium. Rahman and Sultan [8] implemented an efficient, iterative, 
finite difference method to study the thermal radiation interaction of the boundary layer flow of micropolar fluid past a heated vertical porous plate embedded in a porous medium with variable suction as well as heat flux at the plate. Ibrahim et al. [9] evaluated the viscous dissipation and radiation effects on mixed convection flow of micropolar fluid. Sudheer Babu et al. [10] described the effects of mass transfer on unsteady magneto-convection flow of micropolar fluid along a vertical moving porous plate through porous medium with viscous dissipation. Furthermore, detailed reviews of other investigations into radiative heat transfer in micropolar flows are provided by Reddy [11], Olajuwon et al. [12] and Mamatha et al. [13].

In the above investigations, the combined effects of Joule dissipation and chemical reaction in hydromagnetic micropolar transport have been excluded. In most simulations of magnetic heat transfer, the Joule dissipation term is conventionally neglected on the premise that under normal conditions the Eckert number is small based on an order of magnitude analysis, also in many industrial processes e.g. materials fabrication of powders chemical reaction may exert an influential role. These, chemical reactions take place, which may be destructive or constructive in nature and can influence significantly heat and mass diffusion phenomena. Generally, boundary layer flow models utilize first order chemical reaction effects and assume the reaction to be destructive. Rahman [14] studied the effects of viscous dissipation and Joule heating in convective flows of a micropolar fluid, observing that heat transfer rates are decreased with increasing Joule heating effect. Haque et al. [15] examined the steady magnetic natural convection heat transfer in micropolar fluid with Joule heating and viscous dissipation. In numerous process engineering systems, chemical reactions take place, which may be destructive or constructive in nature and can influence significantly heat and mass diffusion phenomena. Generally, boundary layer flow models utilize first order chemical reaction effects and assume the reaction to be destructive. Several investigations have considered reactive heat and mass transfer in external boundary layer flows for micropolar and other fluids. Sheri and Shamshuddin [16] have addressed the problem of coupled heat and mass transfer in magnetohydrodynamic micropolar flow with both viscous dissipation and chemical reaction effects. Sheri and Shamshuddin [17] have further presented finite element numerical solutions for diffusothermal and chemical reaction effects on transient free convection micropolar flow. Recently Ayano and Mathunjwa [18] studied the combined effect of chemical reaction and radiation on micropolar fluid flow over vertical plate with variable temperature. An interesting analysis of exothermic and endothermic kind of chemical reaction over micropolar fluid was investigated by Koriko et al. [19]. Further studies of reactive micropolar flows include Pal and Talukdar [20], Pal and Biswas [21] Modather et al. [22].

In the present article, motivated by simulating nonNewtonian thermal materials processing of powders, we extended the analytical work of Sudheer Babu et al. [10] by taking into account of thermal radiation, Joule dissipation and first order chemical reaction effects and deriving finite element numerical solutions for generalized micropolar radiative-convection flow from a vertical surface in a porous medium. The perturbation approximation form solutions presented by Sudheer Babu et al. [10] provide a benchmark for the present finite element computational solutions. The effects of various emerging thermo-physical parameters on the velocity, micro-rotation (angular) velocity, temperature and concentration profiles as well as on local skin friction coefficient and wall couple stress is visualized and tabulated. The current problem, to the best knowledge of the authors, has not been communicated thusfar in the technical literature.

\section{Mathematical Model}

Free convective flow of an electrically conducting incompressible micro-polar fluid past a vertical plane in the presence of thermal radiation, Joule dissipation and chemical reaction is considered. The plane considered is permeable and is moving with constant velocity $U p$ in a porous medium. The physical configuration is illustrated in Fig. 1. The Eringen [32] micropolar model is employed since it successfully captures microstructural characteristics of complex magnetic polymers i.e. it robustly simulates rotatory motions, gyration of fluid micro-elements. Micropolar fluids can support couple stresses, shear stresses, body couples and, also exhibit microrotational effects and inertia. Darcy's law is assumed and low Reynolds number flow (viscous-dominated regime). A magnetic field of uniform strength $B_{0}$ is applied in a direction parallel to the $y^{\prime}$ axis which is perpendicular to the flow direction. It is assumed that the induced magnetic field is negligible in comparison to the applied magnetic field [23]. Magnetic Reynolds number is very small. The magnetohydrodynamic (MHD) body force term is derived from an order of magnitude analysis of the full Navier-stokes equation. It is also assumed that applied or polarized voltage is neglected so that no energy is added or extracted from the fluid by electrical means. The fluid is considered to be a gray, absorbing-emitting but non-scattering medium and the Rosseland approximation is used to describe the radiative heat flux. The radiative heat flux in the $x^{\prime}$ direction is considered negligible in comparison with that of $y^{\prime}$ direction. Both wall temperature and concentration vary with the distance along the plate and they are always greater than their uniform ambient values existing far from the plate surface. The magnetic micropolar fluid contains a species which is reactive and obeys first order chemical reaction. To simplify the formulation of the boundary conditions, we assumed the size of holes in the porous plate is significantly larger than the characteristic microscopic length scale of the micropolar fluid. It is assumed that the plate is infinite in extent and hence all physical quantities depend only on $y^{\prime}$ and $t^{\prime}$.

Boussinesq approximation is taken in momentum equation. The balances of mass, linear momentum, angular momentum, energy, and concentration species in the Cartesian frame are written as follows: The continuity equation: 


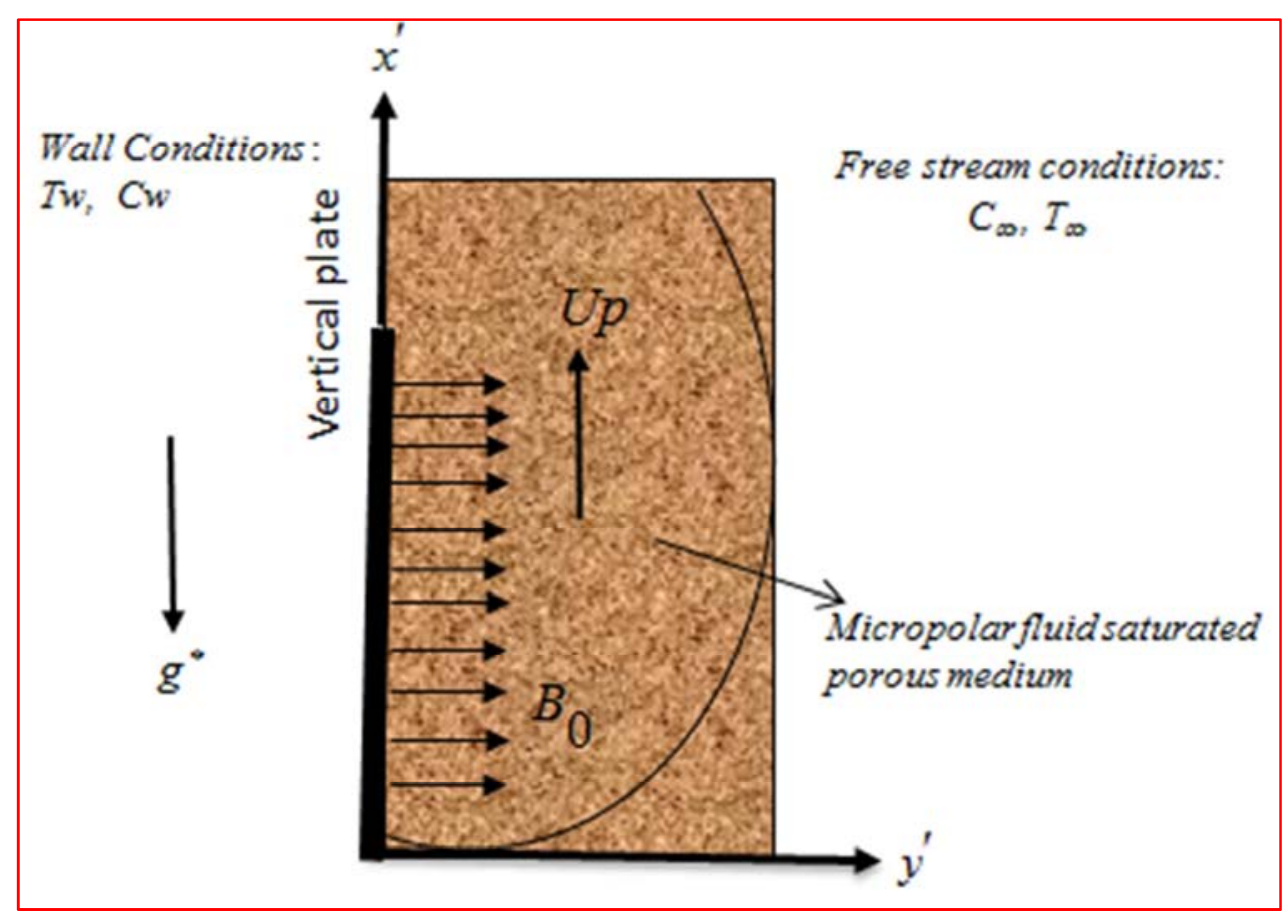

Figure 1. Geometry and coordinate system of the problem.

$$
\frac{\partial v^{\prime}}{\partial y^{\prime}}=0
$$

$$
\begin{gathered}
\frac{\partial u^{\prime}}{\partial t^{\prime}}+v^{\prime} \frac{\partial u^{\prime}}{\partial y^{\prime}}=\left(v+v_{r}\right) \frac{\partial^{2} u^{\prime}}{\partial y^{\prime 2}}+g^{\prime} \beta_{T}\left(T_{w}^{\prime}\right. \\
\left(\frac{\partial N^{\prime}}{\partial t^{\prime}}+v^{\prime} \frac{\partial N^{\prime}}{\partial y^{\prime}}\right)=\frac{\gamma^{\prime}}{\rho j^{\prime}} \frac{\partial^{2} N^{\prime}}{\partial y^{\prime 2}} \\
\frac{\partial T^{\prime}}{\partial y^{\prime}}=\alpha \frac{\partial^{2} T^{\prime}}{\partial y^{\prime 2}}+\frac{1}{\rho C_{p}} \mu\left(\frac{\partial u^{\prime}}{\partial y^{\prime}}\right)^{2}+\frac{\sigma B_{0}^{2}}{\rho C_{p}} u^{\prime 2}-\frac{1}{\rho C_{p}} \frac{\partial q_{r}}{\partial y^{\prime}} \\
\frac{\partial C^{\prime}}{\partial t^{\prime}}+v^{\prime} \frac{\partial C^{\prime}}{\partial y^{\prime}}=D_{m} \frac{\partial^{2} C}{\partial y^{\prime 2}}-K_{C}\left(C_{w}^{\prime}-C_{\infty}^{\prime}\right)
\end{gathered}
$$

Where $u^{\prime}$ and $v^{\prime}$ are the velocity components in $x^{\prime}$ and $y^{\prime}$ axis respectively. $N^{\prime}$ is the micro-rotation component, $v$ is the kinematic viscosity, $v_{r}$ is the kinematic microrotation viscosity, $\rho$ is the constant fluid density, $\sigma$ is the

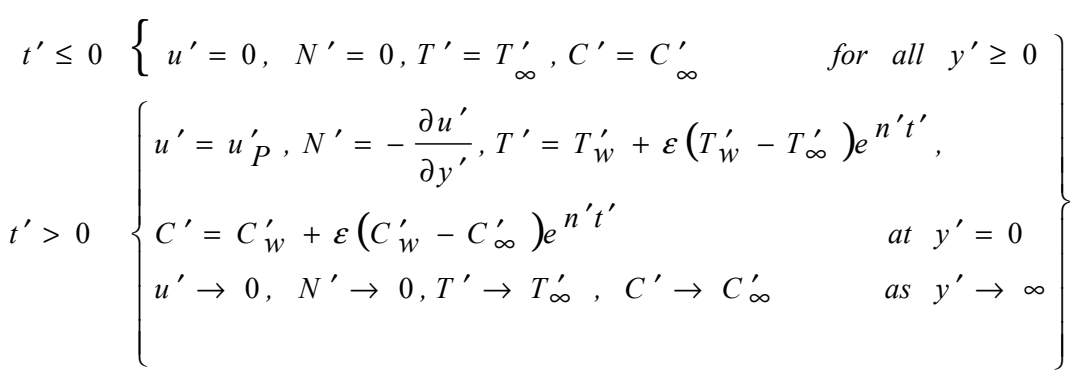


Where $u_{p}^{\prime}$ is plate velocity, it is clear from the equation of continuity that suction velocity normal to the plate is either a constant or a function of time. Hence it is assumed that the suction velocity takes the form:

$$
u^{\prime}=-V_{0}
$$

Where $V_{0}$ is a scale of suction velocity and $V_{0}>0$. The negative sign indicates that the suction velocity is towards the plate. The radiative heat flux term is given by

$$
q_{r}=\frac{-4 \bar{\sigma}}{3 \bar{k}} \frac{\partial T^{\prime 4}}{\partial y^{\prime}}
$$

Here $\bar{\sigma}$ and $\bar{k}$ are the Stefan-Boltzmann constant and mean absorption coefficient respectively. The assumed Rosseland model has been shown to be generally valid for optically-thick fluid media, as considered in viscous fluids [24]. Implementing eqn. (8) results in a highly nonlinear energy equation in $T$ and it is difficult to obtain a solution. However, researchers have resolved this problem by assuming small temperature differences within the fluid flow $[25,26]$. In this situation, Rosseland's model can be linearized about ambient temperature $T_{\infty}^{\prime}$ assuming that the difference in the temperature within the flow is such that $T^{\prime 4}$ can be expressed as a linear combination of the temperature. Using Taylor's series expansion about $T^{\prime}$ the expansion of $T^{\prime 4}$ can be written as follows, neglecting higher order terms:

$$
T^{\prime 4}=T_{\infty}^{\prime 4}+4 T_{\infty}^{\prime 3}\left(T^{\prime}-T_{\infty}^{\prime}\right)+6 T_{\infty}^{\prime 2}\left(T^{\prime}-T_{\infty}^{\prime}\right)^{2}+\ldots \ldots
$$

Neglecting higher order terms beyond the first degree in $\left(T^{\prime}-T_{\infty}^{\prime}\right)$, we have

$$
T^{4} \cong 4 T_{\infty}^{\prime 3} T-3 T_{\infty}^{\prime 4}
$$

Differentiating equation (8) w.r.t $y^{\prime}$ and using (10), we obtain:

$$
\frac{\partial q_{r}}{\partial y^{\prime}}=-\frac{16 T_{\infty}^{\prime 3} \bar{\sigma}}{3 \bar{k}}\left(\frac{\partial^{2} T}{\partial y^{\prime}}\right)
$$

Now simply replacing $T^{\prime 3}$ in Eq. (8) with $T_{\infty}^{\prime 3}$, Eq. (4) can be expressed as follows:

$$
\left(\frac{\partial T^{\prime}}{\partial t^{\prime}}+v^{\prime} \frac{\partial T^{\prime}}{\partial y^{\prime}}\right)=\alpha\left(1+\frac{16 \bar{\sigma}}{3 \bar{k} \kappa} T_{\infty}^{\prime 3}\right) \frac{\partial^{2} T}{\partial y^{\prime 2}}+\frac{\mu}{\rho C_{p}}\left(\frac{\partial u^{\prime}}{\partial y^{\prime}}\right)^{2}+\frac{\sigma B_{0}^{2}}{\rho C_{p}} u^{2}
$$

In order to write the governing equations and boundary conditions in dimensionless form, the following non-dimensional quantities are introduced:

$$
\begin{aligned}
& u=\frac{u^{\prime}}{U_{0}}, \quad v=\frac{v^{\prime}}{V_{0}}, \quad \eta=\frac{V_{0} y^{\prime}}{v}, \quad U_{p}=\frac{u_{p}^{\prime}}{U_{0}}, n=\frac{n^{\prime} v}{V_{o}^{2}}, N=\frac{v}{U_{0} V_{0}} N^{\prime}, \quad t=\frac{t^{\prime} V_{0}^{2}}{v}, \\
& \theta=\frac{T^{\prime}-T_{\infty}^{\prime}}{T_{w}^{\prime}-T_{\infty}^{\prime}}, \varphi=\frac{C^{\prime}-C_{\infty}^{\prime}}{C_{w}^{\prime}-C_{\infty}^{\prime}}, j=\frac{V_{0}^{2}}{v^{2}} j^{\prime}, \gamma^{\prime}=\left(\mu+\frac{\Lambda}{2}\right) j^{\prime}=\mu j^{\prime}\left(1+\frac{\beta}{2}\right), \beta=\frac{\Lambda}{\mu}, \\
& \lambda=\frac{\mu j^{\prime}}{\gamma^{\prime}}=\frac{2}{2+\beta}, M=\frac{\sigma B_{0}^{2} v}{\rho V_{0}^{2}}, K=\frac{V_{0}^{2} K^{\prime}}{v^{2}}, P r=\frac{v}{\alpha}=\frac{\mu C_{p}}{\kappa}, S c=\frac{v}{D_{m}}, K c=\frac{K^{\prime} c v}{V_{0}^{2}} \\
& G r=\frac{v g^{\prime} \beta_{T}\left(T_{w}^{\prime}-T_{\infty}^{\prime}\right)}{U_{0} V_{0}^{2}}, G m=\frac{v g^{\prime} \beta_{C}\left(C_{w}^{\prime}-C_{\infty}^{\prime}\right)}{U_{0} V_{0}^{2}}, R=\frac{16 \bar{\sigma}_{T}^{3}}{3 \kappa \bar{k}}, E c=\frac{V_{0}^{2}}{C_{p}\left(T_{w}^{\prime}-T_{\infty}^{\prime}\right)},
\end{aligned}
$$

$$
\frac{\partial N}{\partial t}-\frac{\partial N}{\partial \eta}=\frac{1}{\lambda}\left(\frac{\partial^{2} N}{\partial \eta^{2}}\right)
$$

$$
\begin{aligned}
& \frac{\partial \theta}{\partial t}-\frac{\partial \theta}{\partial \eta}=\frac{1}{\Gamma} \frac{\partial^{2} \theta}{\partial \eta^{2}}+E c\left(\left(\frac{\partial u}{\partial \eta}\right)^{2}+M u^{2}\right) \\
& \frac{\partial \varphi}{\partial t}-\frac{\partial \varphi}{\partial \eta}=\frac{1}{S c}\left(\frac{\partial^{2} \varphi}{\partial \eta^{2}}\right)-K c \varphi
\end{aligned}
$$$$
\text { Here } \Gamma=\left(1-\frac{4}{3 R+4}\right) \operatorname{Pr}, \quad B=\left(M+\frac{1}{K}\right)
$$ 
Where $\beta, G r, G m, M, K, P_{r}, E c, S c, K c$ denotes the Eringen micropolar vortex viscosity parameter, Grashof number, Modified Grashof number, Magnetic field parameter, Permeability of the porous medium, Prandtl number, Thermal radiation parameter, Heat absorption parameter, Viscous dissipation, Schmidt number and Chemical reaction parameters respectively.

The corresponding initial and boundary conditions in nondimensional form as follow

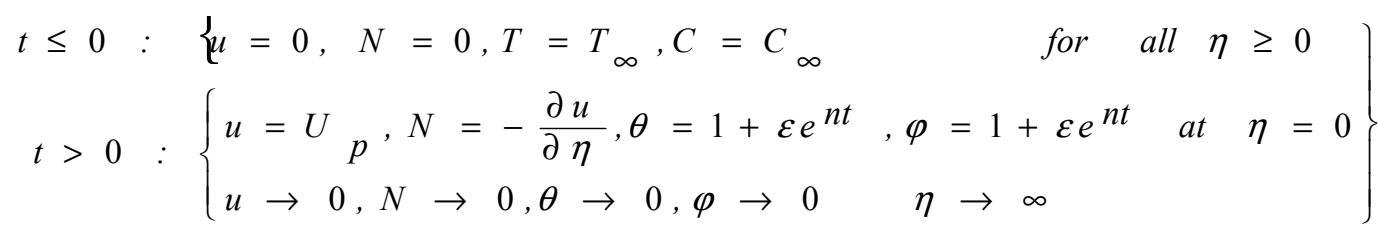

The mathematical statement of the problem is now complete and embodies the solution of Eqs. (14)- (17) with modified boundary conditions (18). The system is wellposed.

\section{Method of Numerical Solution}

The finite element method (FEM) is employed to solve the transformed, coupled boundary value problem defined by eqns. (14) - (17) under (18). FEM is the most popular and adaptable method available to engineers. The general details of the variational finite element method are described at length in Reddy [27] Rao [28] and Bathe [29]. FEM has been applied to study many transport problems of micropolar fluids and magnetic liquids. The fundamental steps involved in the finite-element analysis of a problem are as follows:

Discretization of the infinite fluid domain into finite elements:

The whole domain is divided into a finite number of sub domains, processes known as discretization of the domain. Each sub domain is termed as finite element. The collection of elements is then denoted the finite-element mesh.

Derivation of element equations:

The derivation of finite element equations i.e., algebraic equations among the unknown parameters of the finite element approximation, involves the following three stages.

A typical element is isolated from the mesh and constructing the variational formulation of the differential equation on that typical element.

Assume the form of the approximate solution over a typical finite element.

Derive the finite element equations by substituting the approximate solution into variational formulation.

These steps result in a matrix (known as stiffness matrix) equation of the form $\left.\mid K^{e}\right]\left\{u^{e}\right\}=\left\{F^{e}\right\}$, which defines the finite element model of the original equation.

Assembly of element equations:

The algebraic equations so obtained are assembled by imposing the inter-element continuity conditions (i.e. the values of the nodal variables at the nodes are identical for two or more elements). This yields a large number of algebraic equations known as the global finite element model. This governs the whole flow domain.

Imposition of boundary conditions:

The initial and final boundary conditions defined in equation
(18) are imposed on the above obtained assembled equations.

Solution of assembled equations:

The final matrix equation obtained can be solved by any efficient iterative scheme.

In one-dimensional space, linear and quadratic elements or higher order can be taken. Here the entire flow domain is considered by dividing it into successively sized grids of order $81 \times 81,101 \times 101$ and $121 \times 121$ in the y-axis direction. After many tests a grid size with 101 intervals has been adopted. Thus, all the computations are executed with 101 intervals of equal step size 0.01 . At each node, 4 functions are to be evaluated and after assembly of the element equations, a set of 404 non-linear equations are obtained which necessitate an iterative solution subject to the specified boundary conditions. The iterative process is terminated when the following condition is met: $\sum_{i, j}\left|\xi^{n+1}-\xi^{n}\right| \leq 10^{-6}$ where $\xi=u, N, \theta$ and $\varphi$ are velocity along $x$ axis, microrotation, temperature and concentration respectively. $n$ denote the iterative step. To see the effects of step size (h) the finite element code is run with step sizes as $\mathrm{h}=0.01$ and very good agreement is obtained for different profiles.

The skin friction coefficient, couple stress coefficient, Nusselt number and Sherwood number are important parameters for this type of boundary layer flow and frequently used in materials processing simulations and design.

The skin-friction at the plate in non-dimensional form is given by:

$$
C_{f}=\left(\frac{\partial u}{\partial \eta}\right)_{\eta=0}
$$

The couple stress coefficient at the plate in nondimensional form is given by:

$$
C_{w}=\left(\frac{\partial N}{\partial \eta}\right)_{\eta=0}
$$

Nusselt number is computed in non-dimensional form as:

$$
N u / \operatorname{Re}{ }_{x}=-\left[\frac{\partial \theta}{\partial \eta}\right]_{\eta=0}
$$

Sherwood number is evaluated as in non-dimensional form by: 


$$
S h / \operatorname{Re}_{x}=-\left[\frac{\partial \varphi}{\partial \eta}\right]_{\eta=0}
$$

Here $R e_{x}=\frac{V_{o} x}{v}$ is the local Reynolds number based on the plate suction velocity.

Table 1. Variations of in $C_{f}, C_{w}, N u \operatorname{Re}_{x}^{-1}$ and $\operatorname{Sh} \operatorname{Re}_{x}^{-1}$ for different $\beta, G r$, Gm and $M$.

\begin{tabular}{llllllll}
\hline $\boldsymbol{\beta}$ & $\boldsymbol{G} \boldsymbol{r}$ & $\boldsymbol{G} \boldsymbol{M}$ & $\boldsymbol{M}$ & $\boldsymbol{C}_{\boldsymbol{f}}$ & $\boldsymbol{C}_{\boldsymbol{w}}$ & $N u \operatorname{Re}_{x}^{-1}$ & $S h R e^{-1}$ \\
\hline 0.0 & 2.0 & 2.0 & 2.0 & 3.4848 & 3.4789 & 0.5372 & 0.7113 \\
0.1 & 2.0 & 2.0 & 2.0 & 0.7551 & 0.7550 & 0.5375 & 0.7113 \\
0.5 & 2.0 & 2.0 & 2.0 & 0.7097 & 0.7096 & 0.5310 & 0.7113 \\
0.1 & 4.0 & 2.0 & 2.0 & 1.7222 & 1.7129 & 0.5345 & 0.7113 \\
0.1 & 2.0 & 4.0 & 2.0 & 1.7174 & 1.7115 & 0.5050 & 0.7113 \\
0.1 & 2.0 & 2.0 & 1.0 & 1.1444 & 1.1445 & 0.5368 & 0.7113 \\
\hline
\end{tabular}

Table 2. Variations of in $C_{f}, C_{w}, N u \operatorname{Re}_{x}^{-1}$ and $S h \operatorname{Re}_{x}^{-1}$ for different Pr, R, Ec and Sc.

\begin{tabular}{llllllll}
\hline $\operatorname{Pr}$ & $\boldsymbol{R}$ & $\boldsymbol{E} \boldsymbol{c}$ & $\boldsymbol{S} \boldsymbol{c}$ & $\boldsymbol{C}_{\boldsymbol{f}}$ & $\boldsymbol{C}_{\boldsymbol{w}}$ & $N_{u} \operatorname{Re}_{x}^{-1}$ & \multicolumn{2}{c}{$\operatorname{Re}^{-1}$} \\
\hline 0.71 & 2.0 & 0.01 & 0.6 & 3.4847 & 3.7937 & 0.5372 & 0.7113 \\
5.0 & 2.0 & 0.01 & 0.6 & 0.6873 & 0.6372 & 0.6722 & 0.7113 \\
0.71 & 1.0 & 0.01 & 0.6 & 0.7845 & 0.7844 & 0.4151 & 0.7113 \\
0.71 & 2.0 & 1.0 & 0.6 & 1.6181 & 0.6180 & 0.7116 & 0.7113 \\
0.71 & 2.0 & 0.01 & 0.2 & 0.9494 & 0.9493 & 0.5390 & 0.3109 \\
\hline
\end{tabular}

Table 3. Effects of $K c$ on $C_{f}, C_{w}$, Nu Re ${ }_{x}^{-1}$ and Sh $\operatorname{Re}_{x}^{-1}$ for with $t=1, n=0.1, \varepsilon=0.001 U p=0.5, \beta=0.5, M=2, G r=G m=2, P r=0.71, R=2, E c=0.01$.

\begin{tabular}{|c|c|c|c|c|c|}
\hline parameter & values & $c_{f}$ & $C_{w}$ & $N u \operatorname{Re}_{x}^{-1}$ & $\operatorname{Sh} \operatorname{Re}_{x}^{-1}$ \\
\hline $\mathrm{Kc}$ & $\begin{array}{l}1.0 \\
2.0\end{array}$ & $\begin{array}{l}2.4215 \\
2.3312\end{array}$ & $\begin{array}{l}2.7313 \\
2.7215\end{array}$ & $\begin{array}{l}0.5663 \\
0.5633 \\
\end{array}$ & $\begin{array}{l}1.4615 \\
1.4821\end{array}$ \\
\hline
\end{tabular}

Table 1 and Table 2 document the friction factor, surface heat transfer and mass transfer rate dependency on the emerging thermo-physical parameters. Table 1 depicts the effect of $\beta, G r, G m, M \quad$ on $\quad C_{f}, C_{w}, N u / R e_{x}, S h / R e_{x}$ respectively. It is observed that the skin friction decreases as $\beta$, $G m$ increases while it increases as $G r$ increases. As $M$ decreases skin friction increases. The same trend is observed in case of wall couple stress. Further, it is observed that the Nusselt number decreases as $\beta, G r, G m$ increases but as $M$ increases, Nusselt number decreases. Sherwood number has no significant effect on $\beta, G r, G m, M$. Table 2 depicts the effect of $\operatorname{Pr}, R, E c, S c \quad$ on $\quad C_{f}, C_{w}, N u / R e_{x}, S h / R e_{x}$ respectively. The skin friction coefficient decreases as $\operatorname{Pr}, E c, R$ increases, while it decreases as $S c$ decreases. The same trend is computed in the case of wall couple stress (wall micro-rotation gradient). Further, it is observed that the

Nusselt number decreases as $R$ decreases. Nusselt number increases as $S c$ decreases, while it increases as $P r, E c$ increases. Sherwood number decreases as $S c$ decreases No tangible modification is computed in Sherwood number (wall mass transfer rate) with a change in $\operatorname{Pr}, E c, R$. Numerical values of the coefficients proportional to the skin friction
$C_{f}$, couple stress coefficient $C_{w}$, Nusselt number $N u$ and Sherwood number $S h$ are given in Table 3 for the general model with all parameters invoked. It is evident from Table 3 homogeneous chemical reaction parameter $K c$ increase, the skin friction coefficient $C_{f}$ and wall couple stress coefficient $C_{w}$ both decrease. Also, it is apparent that as chemical reaction $K c$ increases, a significant increase is computed in Sherwood number, $S h$.

\section{Results and Interpretation}

The nonlinear boundary value problem solved in the previous section is dictated by an extensive number of thermal and hydrodynamic parameters. In order to gain a clear insight into the physical problem, numerical calculations for distribution of the velocity, microrotation (angular) velocity, temperature and concentration for different values of these parameters is conducted with graphical illustrations (Figures 2-17). For the purpose of our computations, we adopted the following default parameters: $\mathcal{E}=0.001, \quad n=0.1, \quad t=1, \quad U_{p}=0.5, \quad \beta=0.1, \quad G r=2$, 
$G m=2, \quad M=2, \quad K=1, \quad P r=0.71, \quad S c=0.6, \quad R=1.0$, $E c=0.01, K c=1.0$ and all graphs therefore correspond to these values unless specifically indicated otherwise on the appropriate graph. The permeability in all the figures plotted is set at 0.5 which corresponds to a highly porous regime, characteristic of certain materials processing systems. The value of $\mathrm{Pr}$ is taken to be 0.71 which corresponds to air at $20^{\circ} \mathrm{C}$ and 1 atmospheric pressure and the value of Sc is 0.6 (water-vapour). Numerical values of the coefficients proportional to the skin friction $C_{f}$, couple stress proportional to the skin friction $C_{f}$, couple stress coefficient $C_{w}$, Nusselt number $\mathrm{Nu}$ and Sherwood number $\mathrm{Sh}$ are given in Table 1, Table 2 and Table 3 for the general model with all parameters invoked.

Figures 2-3 illustrate the response of microrotation velocity parameter $\beta$ on velocity and micro-rotation. It is seen that as $\beta$ increases, the velocity gradient near the porous plate decreases, and then approaches to the free stream velocity. Also, it is noteworthy that velocity distribution across the boundary layer is lower for Newtonian fluid ( $\beta=$ 0 ) as compared with stronger micropolar fluid $(\beta=0.2)$ for the same conditions and fluid properties. Micropolarity (i.e. increasing vortex viscosity of micro-elements) therefore consistently induces deceleration in the flow adjacent to the plate. All profiles are parabolic and peak at some distance from the wall, decaying smoothly to vanish in the free stream. In addition, the magnitude of microrotation at the wall is decreased as $\beta$ increases. However, the distribution of microrotation across boundary layer does not show consistent variations with increase of $\beta$.

The effect of thermal radiation-conduction parameter $(R)$ on temperature is presented in Figure 4. This parameter is defined as $R=16 \bar{\sigma} T_{\infty}^{3} / 3 k \bar{k}$ and features in the augmented thermal diffusion term in eqn. (16) i.e. $1 / \Gamma\left(\partial^{2} \theta / \partial y^{2}\right)$. It defines the relative contribution of thermal radiation heat transfer to thermal conduction heat transfer. When $R<1$ thermal conduction dominates. When $R=1$ both thermal conduction and thermal radiation contributions are equal. For $R>1$ thermal radiation dominates over thermal conduction. In the present simulations, we confine attention to the last of these three cases i.e. $R>1$ wherein thermal radiative flux is substantial. Increasing radiation-conduction parameter is found to decrease temperatures in the boundary layer. Thermal boundary layer thickness is therefore reduced with greater values of $R$.

Figure 5 represent the influence of chemical reaction parameter $(K c)$ on concentration profiles. The reaction parameter is based on a first-order irreversible chemical reaction which takes place both in the bulk of the fluid (homogeneous) as well as at the plate which is assumed to be catalytic to chemical reaction. We consider the destructive type of homogenous chemical reaction. It is noticed that concentration distributions decrease when the chemical reaction increases. Physically, for a destructive case, chemical reaction takes place and progressively destroys the original species. This, in turn, suppresses molecular diffusion of the remaining species which leads to a fall in concentration magnitudes and a decrease in concentration boundary layer thickness.

Figure 6 illustrates the influence of Prandtl number $(P r)$ on temperature profiles. Prandtl number represents the relative rate of momentum diffusion to energy diffusion. With $\mathrm{Pr}>1$ the momentum diffusion rate also exceeds the thermal diffusion rate in the fluid. It is observed that there is a strong depression in temperature with greater Prandtl number $(P r)$, greater Prandtl number corresponds to a lower thermal conductivity. This leads to a reduction in thermal energy convected through the fluid from the plate $(G r>0$ i.e. plate cooling) and depresses the thermal boundary layer thickness.

Figure 7 present the effects of the viscous dissipation parameter i.e., the Eckert number $E c$ on temperature field. Eckert number signifies the quantity of mechanical energy converted via internal friction to thermal energy i.e. heat dissipation. Increasing $E c$ values will therefore cause an increase in thermal energy contributing to the flow and will heat the regime. Positive Eckert number implies cooling of the wall and therefore a transfer of heat to the micropolar fluid, also convection is enhanced. Temperatures are markedly increased with greater Eckert number. For all nonzero values of $E c$ the temperature overshoot near the wall is distinct; this overshoot migrates marginally further into the boundary layer with an increase in $E c$. Very smooth decays in temperature profiles are observed for all values of Eckert number and the convergence of profiles in the free stream indicates that an adequately large infinity boundary condition has been imposed in the finite element model.

The profiles of the velocity and microrotation in the boundary layer for various values of the plate moving velocity $U_{p}$ are shown in Figures $8-9$ in the direction of the fluid flow. It is noticed that the peak value of velocity across the boundary layer increases near the porous plate as the plate velocity increases. The results also show that the magnitude of microrotation on porous plate decreases as $U_{p}$ increases. The linear flow is therefore accelerated with greater plate velocity whereas the micro-rotation (angular flow) of micro-elements is inhibited i.e. decelerated.

Figures 10-13 represents the influence of Grashof number $G r$ and modified Grashof number $G m$ on velocity and microrotation profiles. The thermal Grashof number, $G r$, quantifies the relative magnitude of the buoyancy force and the opposing frictional (viscous) forces acting on the micropolar fluid. Physically the positive, negative and zero (i.e., $G r>0, G r<0$ and $G r=0$ ) values of the Grashof number correspond to cooling, heating of the boundary surface and absence of free convection currents, respectively. The species (solutal) Grashof number i.e. $G m$ embodies the relative contribution of species buoyancy force to viscous hydrodynamic force. It is observed that the velocity increases as $G r$ or $G m$ increases. Furthermore, the peak value of velocity increases rapidly near the wall of the porous plate. However, the converse behavior is computed in the case of micro-rotation profiles. Thermal and species buoyancy 
therefore modify linear and angular velocity fields in a different fashion with different implications for boundary layer thicknesses.

Figures 14-15 show the pattern of the velocity and microrotation for different values of magnetic field parameter $M$. It is observed that the amplitude of the velocity as well as the boundary layer thickness decreases when $M$ is increased. Physically, in magnetohydrodynamic materials processing, the applied magnetic field exerts a retarding effect on the free convective flow, transverse to the direction of imposition of the magnetic field. With increasing the values of $M$, this type of resisting force slows down the fluid i.e. with stronger magnetic field strength the flow is decelerated and this is confirmed with the decreasing velocity distribution across the boundary layer. In case of figure 15 an increase in magnetic parameter is observed to significantly accelerate the angular velocity i.e. enhance the magnitude of micro-rotation, although the effect is more localized at the plate surface and progressively grows further from the plate. In both figures 14 and 15 asymptotically smooth solutions are obtained indicating that a sufficiently large infinity boundary condition is prescribed in the free stream. Linear momentum boundary layer thickness is therefore increased with greater magnetic parameter whereas angular momentum boundary layer thickness is reduced.

Figures 16-17 visualize the effect of the porous medium permeability parameter $(K)$ on both velocity and microrotation fields. This parameter characterizes the hydraulic transmissivity of the porous medium. With increasing permeability, the regime, the quantity of solid fibers progressively decreases. The Darcian bulk impedance to flow is therefore also decreased. This results in acceleration in the velocity $u$, as observed in Fig. 16. This behaviour is sustained across the boundary layer i.e. for all values of transverse coordinate, $\eta$. It is also apparent that micro-rotation i.e. angular velocity is enhanced with greater permeability parameter although the effect is more prominent near the plate surface and is weakened with further distance into the boundary layer. Since the permeability parameter does not arise in the angular momentum conservation (boundary layer) eqn. (15) the accelerating effect on micro-rotation is sustained via the boost in linear momentum experienced through the coupling terms which link both linear and angular momentum fields. The increase in permeability implies greater void space in the porous medium. This allows an enhancement in gyratory motions as the micro-elements are afforded greater space in which to spin.

The concentration profiles for different values of Schmidt number, $S c$ are illustrated in Figures 18. The Schmidt number embodies the ratio of the momentum to the mass diffusivity i.e. $S c=v / D$. The Schmidt number therefore quantifies the relative effectiveness of momentum and mass transport by diffusion in the hydrodynamic (velocity) and concentration (species) boundary layers. For $S c>1$ momentum diffusion rate exceeds the species diffusion rate. The opposite applies for $S c<1$. For $S c=1$ both momentum and concentration (species) boundary layers will have the same thickness and diffusivity rates will be equal. However, it is apparent that species (concentration) profiles gradually increase with higher Schmidt number. Smaller values of $S c$ are equivalent to increasing the chemical molecular diffusivity and vice versa for larger values of $S c$.

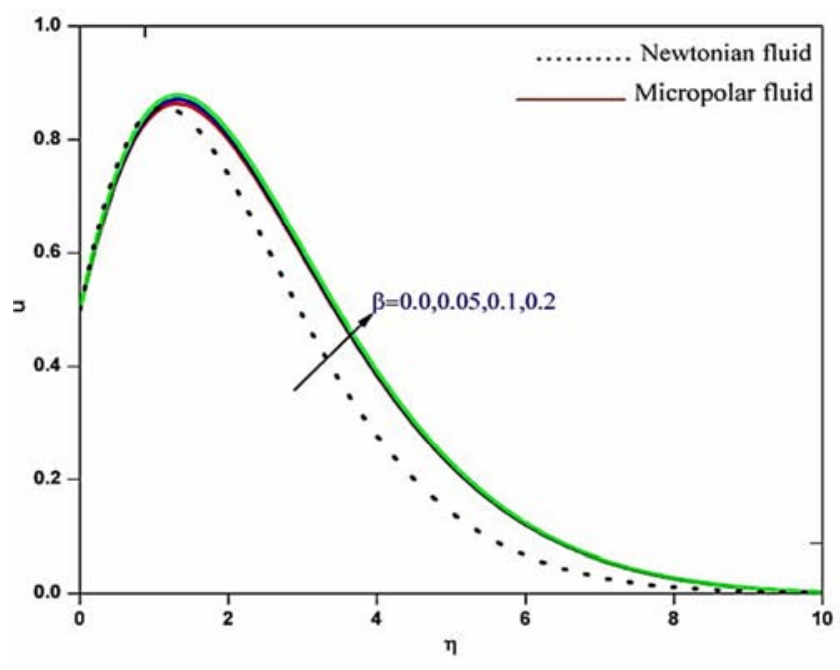

Figure 2. Velocity profiles for variou values of $\beta$.

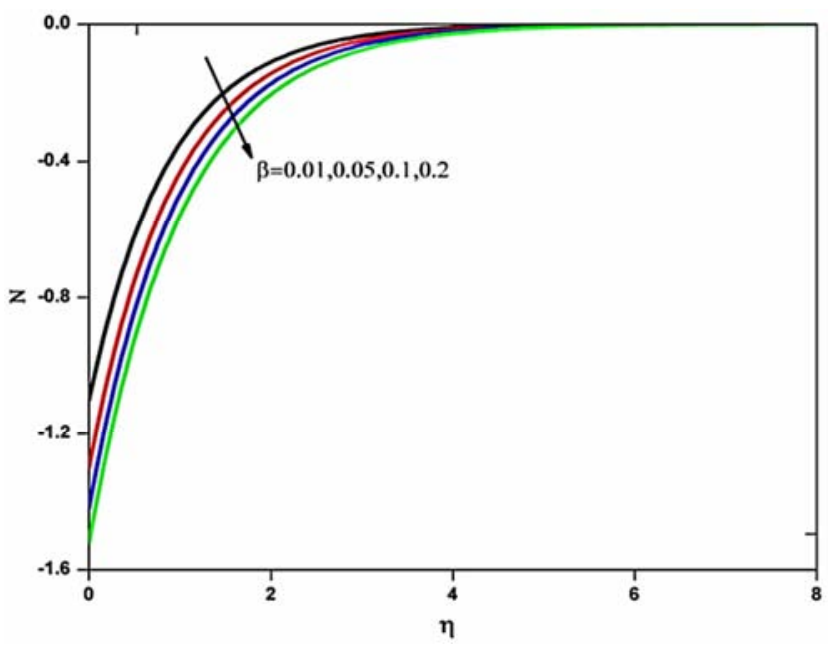

Figure 3. Microrotation profiles for various values of $\beta$.

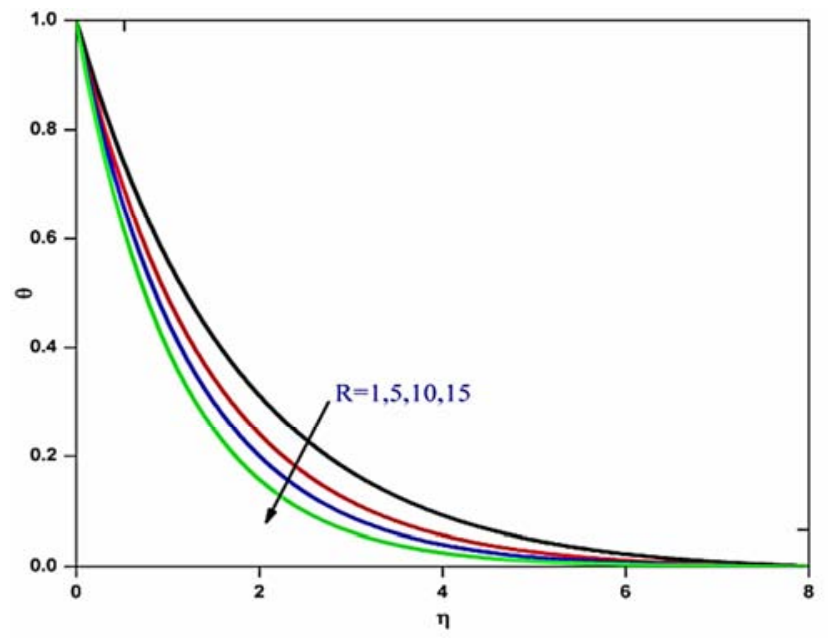

Figure 4. Temperature profiles for various values of $R$. 


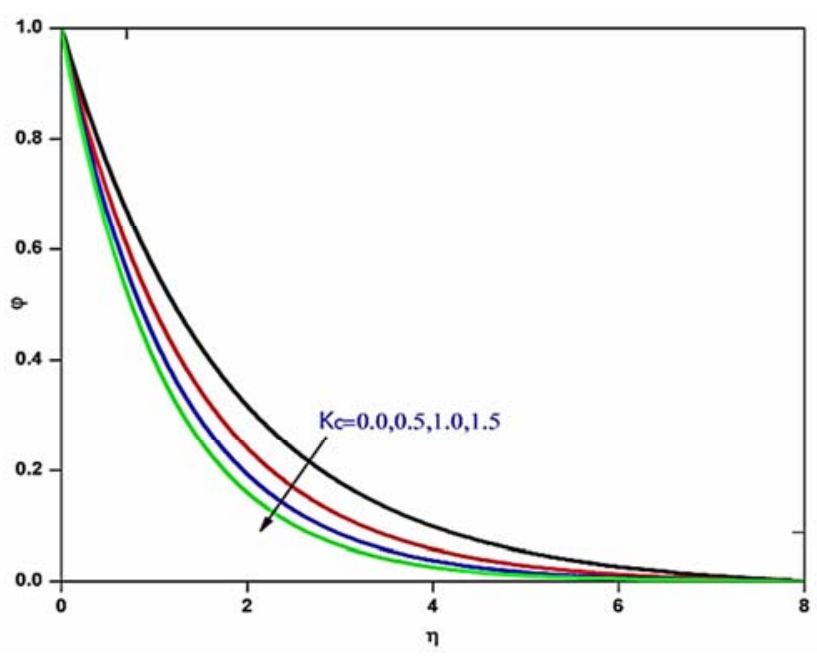

Figure 5. Concentration profiles for various values of $K c$.

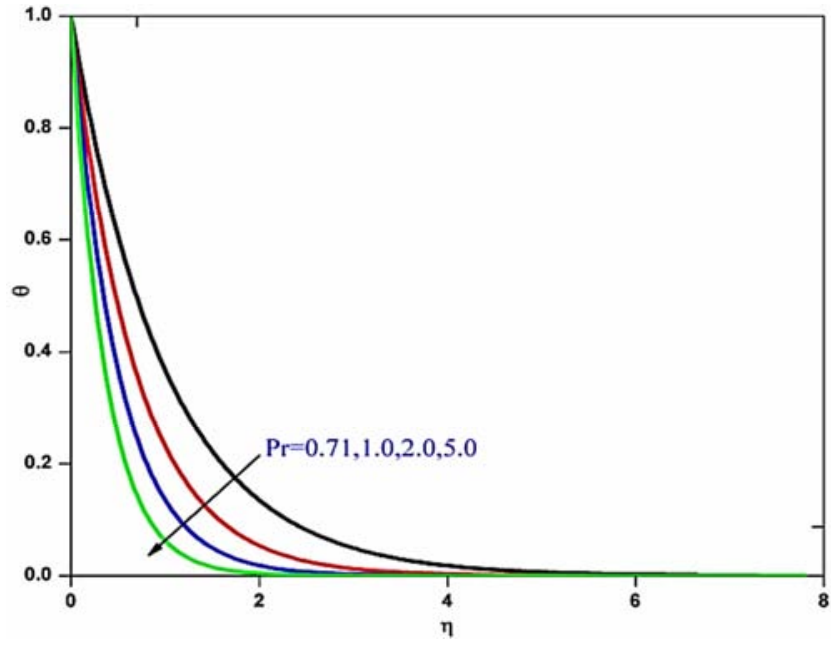

Figure 6. Temperature profiles for various values of $\mathrm{Pr}$.

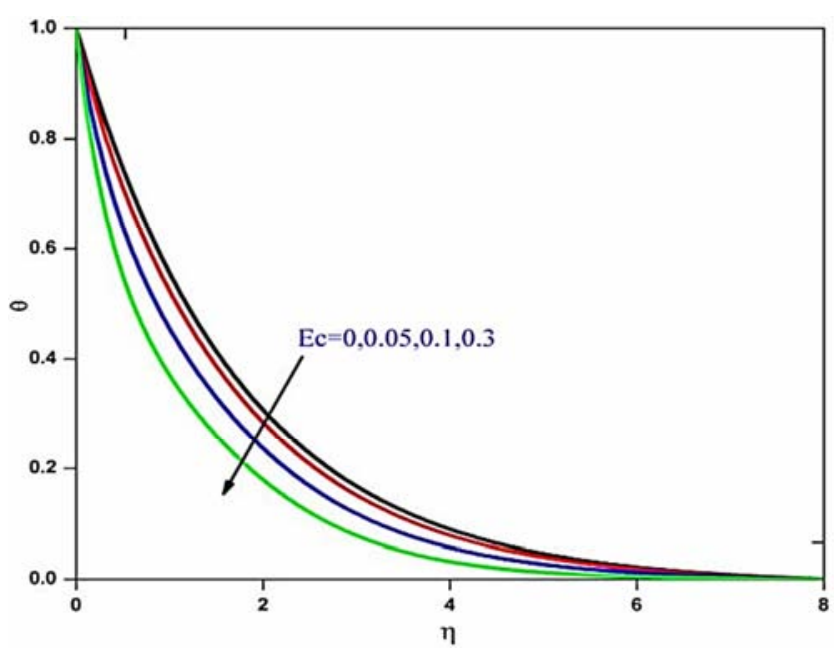

Figure 7. Temperature profiles for various values of $E c$.

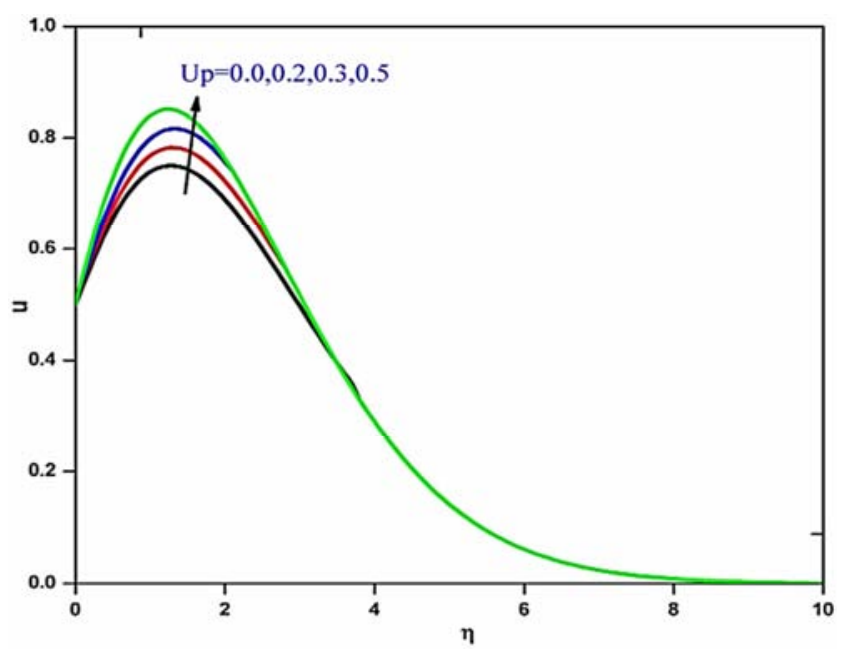

Figure 8. Velocity profiles for various values of $U_{p}$.

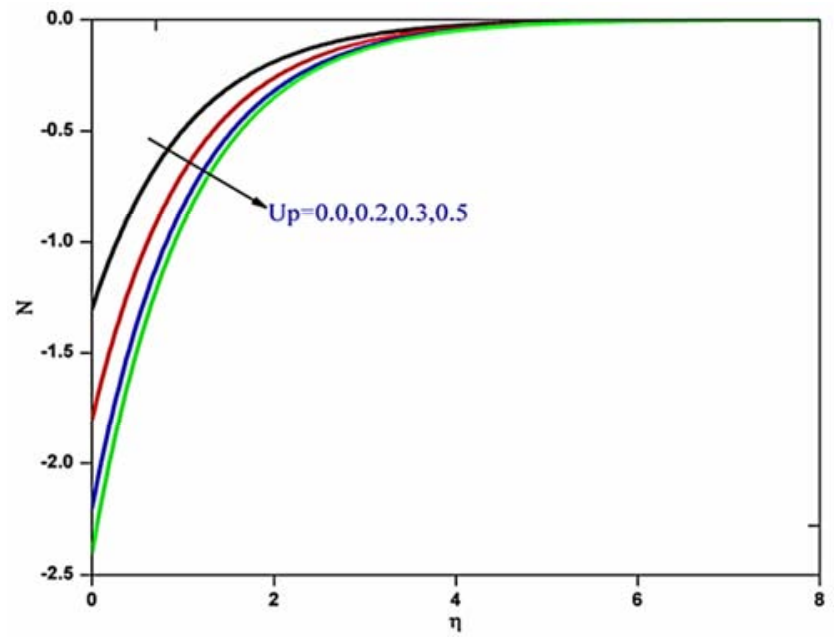

Figure 9. Microrotation profiles for various value $U_{p}$.

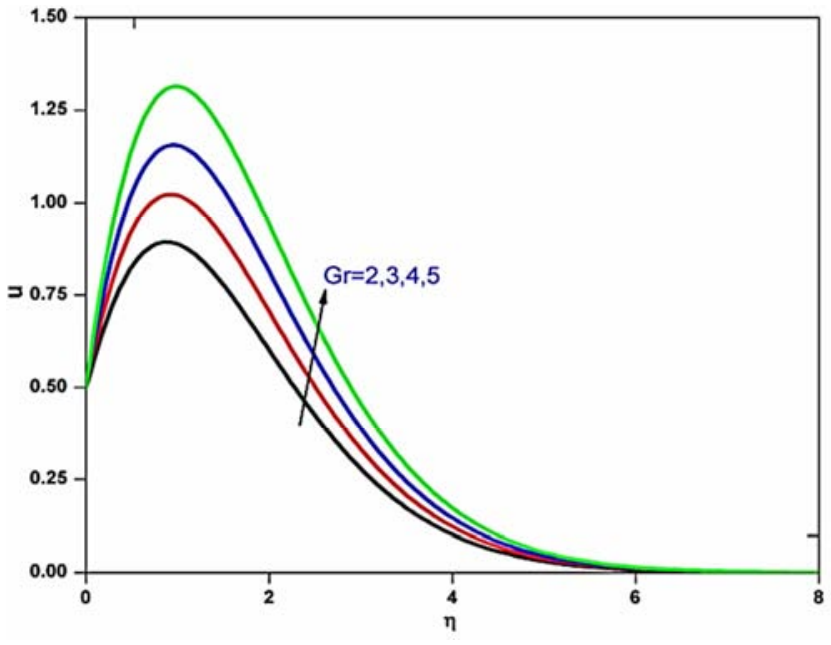

Figure 10. Velocity profiles for various values of $G r$ 


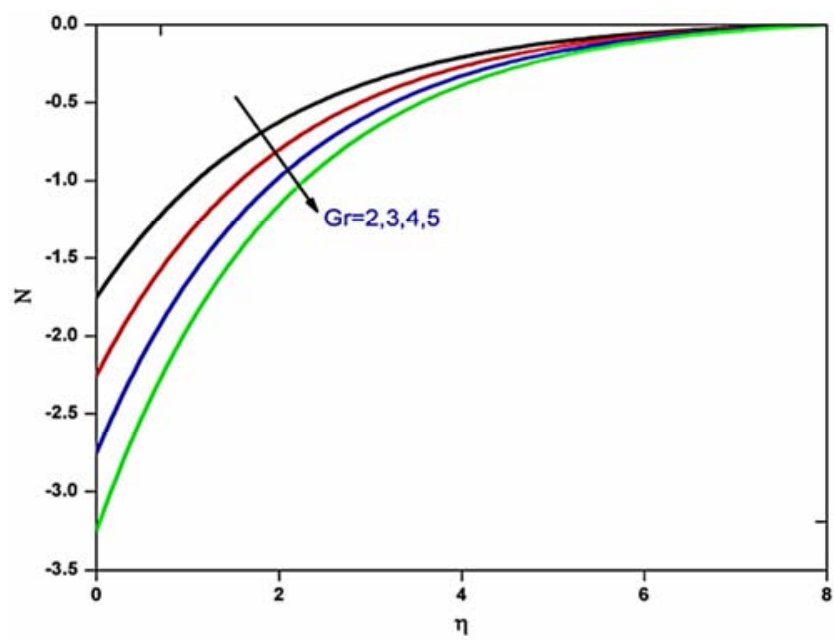

Figure 11. Microrotation profiles for various values of $G r$.

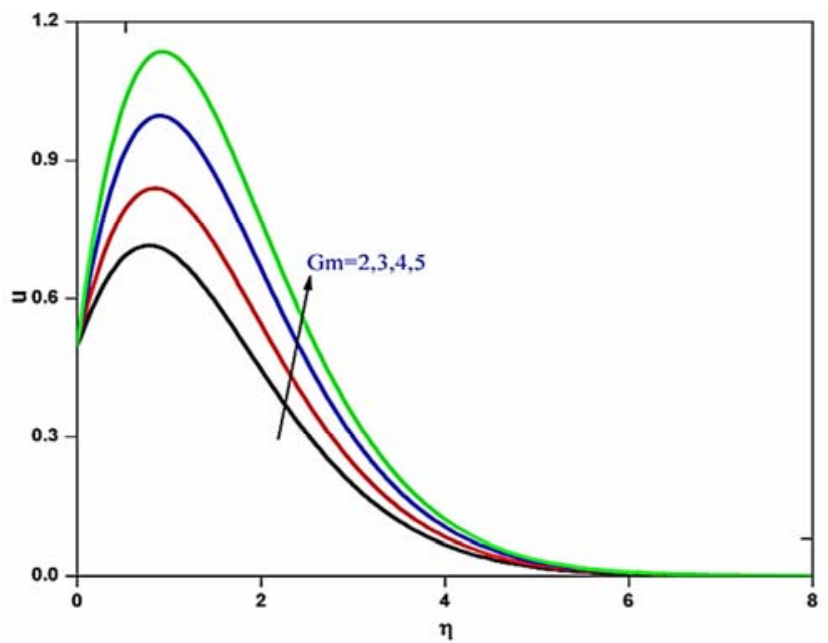

Figure 12. Velocity profiles for various values of $\mathrm{Gm}$.

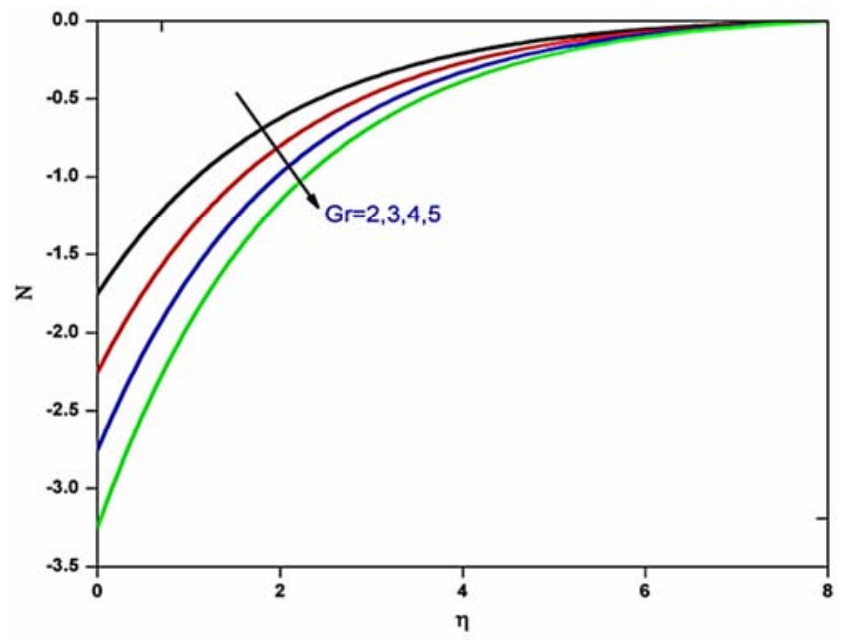

Figure 13. Microrotation profiles for various values of $G r$.

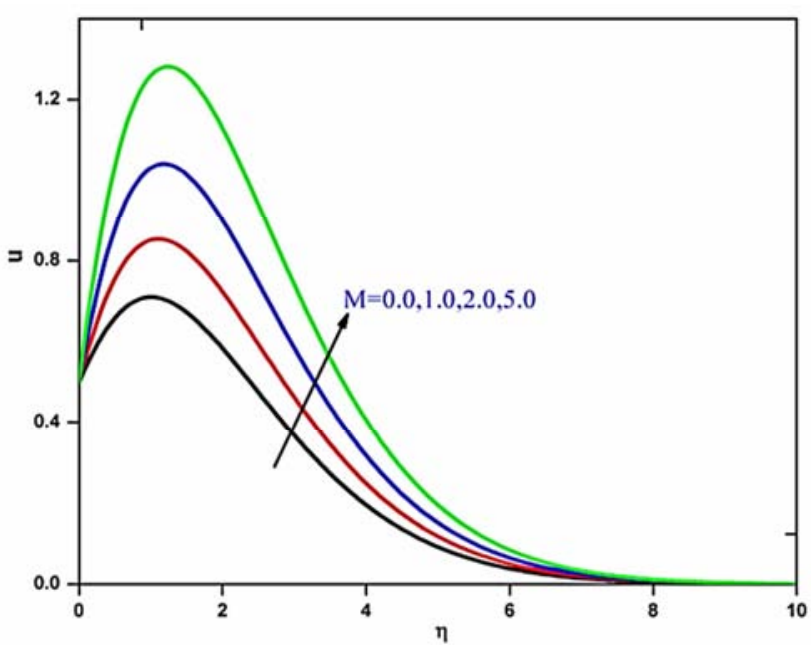

Figure 14. velocity profiles for Various values of $M$.

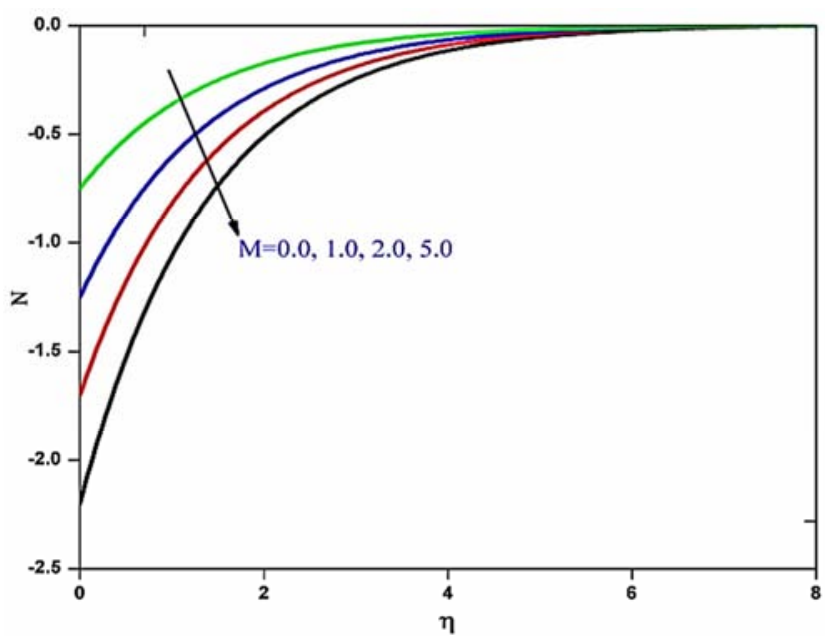

Figure 15. Microrotation profiles for various values of $M$.

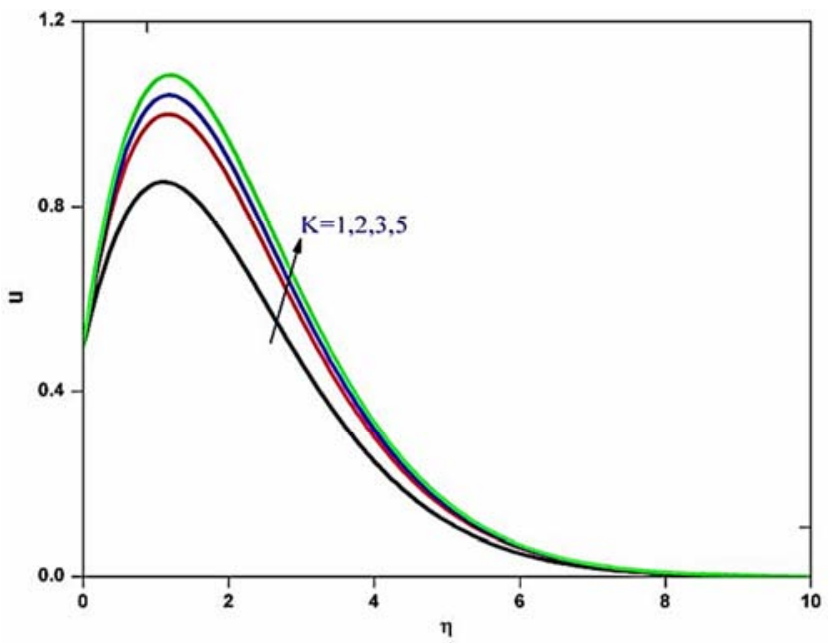

Figure 16. Velocity profiles for various values of $K$. 


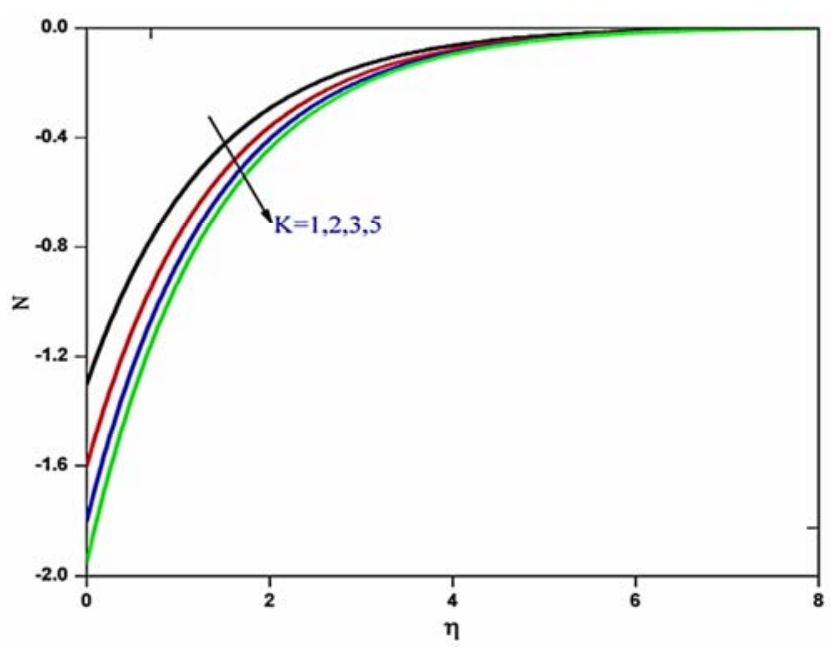

Figure 17. Microrotation profiles for various values of $K$.

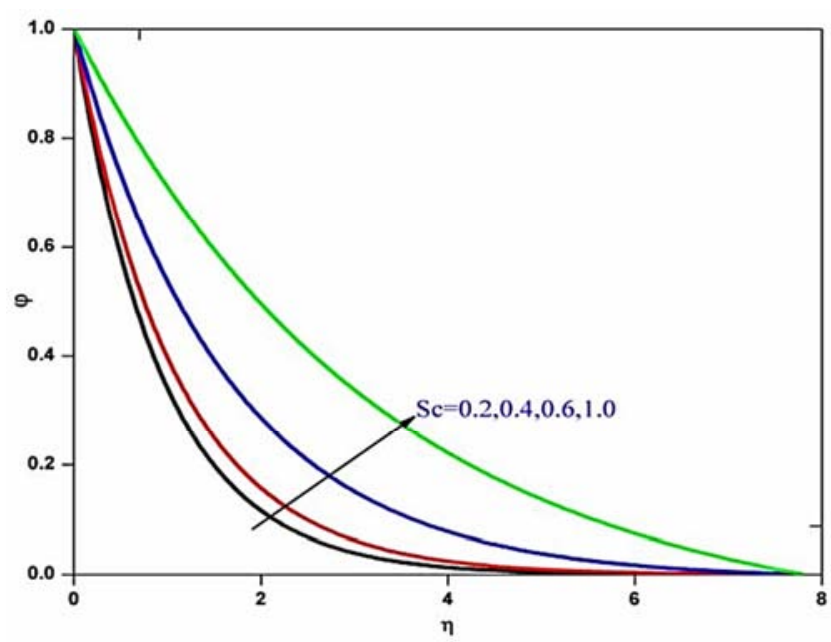

Figure 18. Concentration profiles for various values of $S c$.

\section{Conclusions}

A mathematical model has been presented for radiative magnetic free convection heat and mass in transient flow of an incompressible, micropolar fluid from an inclined plate in porous media. Heat source/sink and homogeneous chemical reaction effects have been included in the formulation. The conservation equations for momentum, angular momentum (micro-rotation component), energy and concentration have been non-dimensionlized with appropriate variables. The resulting non-linear, transient, coupled system of partial differential equations and set of initial and boundary conditions has been solved numerically, using the variational finite element method with a Galerkin weighted residual scheme. Computations have been executed in MATLAB software, and have shown that the flow is accelerated and momentum boundary layer thickness decreased with increasing values of $U_{p}, G r, G m, K$ and Ec but in case of $\beta$, $\mathrm{M}, \mathrm{R}, \mathrm{Sc}$ and $\mathrm{Kc}$ the flow is decelerated and momentum boundary layer thickness increased. Angular velocity (Microrotation) is suppressed as $\beta, \mathrm{U}_{\mathrm{p}}, \mathrm{Gr}, \mathrm{Gm}$, and $\mathrm{K}$ increases, conversely angular velocity is elevated with $\mathrm{M}$ increases. Increasing Eckert number Ec elevates temperature and enhances thickness of thermal boundary layer. Increasing Schmidt number elevates concentration and enhances the thickness of the species boundary layer. Increasing homogeneous chemical reaction parameter decreases concentration and reduces concentration boundary layer thickness. Sherwood number (wall mass transfer rate) is enhanced with increasing permeability and homogeneous chemical reaction.

The finite element code developed has resolved efficiently the nonlinear micropolar transport phenomena in vertical plate magnetohydrodynamic heat and mass transfer. Future studies will consider magnetic induction effects and will be reported soon.

\section{Acknowledgement}

The author gratefully acknowledges the referees for their constructive comments which improved the quality of the paper.

\section{References}

[1] A. C. Eringen, Theory of micropolar fluids, J. Appl. Math. Mech., Vol 16, 1996, pp. 1-18.

[2] A. C. Eringen, Micro-continuum field theories II Fluent media, Springer, Newyork (2001).

[3] G. Lukaszewicz, Micropolar Fluids, Modelling and Simulation, Birkhauser Boston, Boston, (1999).

[4] T. Ariman, M. A. Turk, N. D. Sylvester, Applications of Micro continuum fluid mechanics-a review, Int. J. Eng. Sci. Vol.11, 1973, pp. 905-930.

[5] T. Ariman, M. A. Turk, N. D. Sylvester, Applications of Micro continuum fluid mechanics-a review, Int. J. Eng. Sci. Vol.12, 1974, pp. 273-293.

[6] A. Raptis, C. Perdikis, Viscoelastic flow by the presence of radiation. ZAMP, Vol. 78, 1998, pp. 277-279.

[7] E. M. Abo-Eldahab, A. F. Ghonaim, Radiation effect on heat transfer of a micropolar fluid through a porous medium, App. Math. Comput, Vol.169, No.1, 2005, pp. 500-516.

[8] M. M. Rahman, Y. Sultana, Radiative heat transfer flow of micropolar fluid with variable heat flux in porous medium, Nonlinear Analysis, Modelling and Control, Vol. 13, 2008, pp. 71-87.

[9] F. S. Ibrahim, A. M. Elaiw, A. A. Bakr, Influence of viscous dissiption and radiation on unsteady MHD mixed convection flow of micropolar fluids. Applied Mathematics \& information Sciences-An International Journal, Vol. 2, No. 2, 2008, pp. 143-162.

[10] M. Sudheer Babu, J. Girish Kumar, T. Shankar reddy, Mass transfer effects on unsteady MHD convective flow of a micropolar fluid past a vertical moving porous plate through porous medium with viscous dissipation. Int. J. Appl. Math. Mech., Vol. 9, No.6, 2013, pp. 48-67 
[11] M. G. Reddy, Mass transfer effects on the insteady MHD radiative-convective flow of a micropolar fluid past a vertical porous plate with variable heat and mass flux, Journal of Engineering Physics and Thermophysics, Vol. 86, No. 2, 2013, pp. 406-415.

[12] B. I. Olajuwon, J. I. Oahimire, M. Ferdow, Effect of Thermal radiation and Hall current on heat and mass transfer of unsteady MHD flow of a viscoelastic micropolar fluid through a porous medium. Engineering Science \& Technology-An International Journal, Vol. 17, 2014, pp. 185-193.

[13] B. Mamatha, M. C. Raju, S. V. K. Varma, Thermal diffusion effect on MHD mixed convection unsteady flow of micropolar fluid past a semi-infinite vertical porous plate with radiation and mass transfer. Int. J. Eng. Reaserch in Africa, Vol. 13, 2015, pp. 21-37.

[14] M. M. Rahman, Convective flows of micropolar fluids from radiative isothermal porous surfaces with viscous dissipation and Joule heating, Commun. Nonlinear. Sci. Simulat. Vol. 14, 2009, pp. 3018-3030.

[15] MD. Ziaul Haque, MD. Mahmud Alam, M. Ferdows, A. Postelnicu, Micropolar fluid behaviours on steady MHD free convection and mass transfer flow with constant heat and mass fluxes, Joule heating and viscous dissipation, Journal of King Saud University-Engineering Sciences, Vol. 24, 2012, pp. 71-84.

[16] S. Siva Reddy, MD. Shamshuddin, Heat and mass transfer on the MHD flow of a micropolar fluid in the presence of viscous dissipation and chemical reaction, Procedia Eng., Vol. 127, 2015, pp. 885-892.

[17] S. Siva Reddy, MD. Shamshuddin, Diffusion-thermo and chemical reaction effects on an unsteady MHD free convection flow in a micropolar fluid. Theoretical and Applied Mechanics, Vol. 43, 2016, pp. 117-131.

[18] M. S. Ayano, J. S. Mathunjwa, Chemical reaction and radiation effects on unsteady MHD micropolar fluid flow over a vertical plate with variable temperature. Frontiers in Heat and Mass Transfer Vol. 7, No.1, 2016. doi: 10.5098/hmt.7.9.

[19] O. K. Koriko, A. J. Omowaye, I. L. Animasaun, I. O. Babatunde, Boundary layer analysis of exothermic and endothermic kind of chemical reaction in the flow of nonDarcian unsteady micropolar fluid along an infinite vertical surface. Int. J. Eng. Reaserch in Africa, Vol. 28, 2017, pp. 90101. doi: 10.4028/www.scientific.net/JERA.28-90.

[20] D. Pal, B. Talukdar, Perturbation technique for un-steady MHD mixed convection periodic flow, heat and mass transfer in micropolar fluid with chemical reaction in the presence of thermal radiation, Central European J. Physics, Vol. 10, 2012, pp. $1150-1167$.
[21] D. Pal, S. Biswas, Perturbation analysis of magnetohydrodynamics oscillatory flow on convective-radiative heat and mass transfer of micropolar fluid in a porous medium with chemical reaction, Engineering Science and Technology: An International Journal, Vol. 19, 2016, pp. 444-462.

[22] M. Modather, A. M. Rashad, A. J. Chamkha, An analytical study of MHD heat and mass transfer oscillatory flow of a micropolar fluid over a vertical permeable plate in porous medium, Turkish Journal of Engineering and Environmental Sciences, Vol. 33, No.4, 2009, pp. 245-257.

[23] T. G. Cowling, Magnetohydrodynamics, Wiley Inter Science, Newyork (1957).

[24] Bég, O. Anwar, Numerical methods for multi-physical magnetohydrodynamics, New developments in Hydrodynamics Reaserch, Maximiano J. Ibragimov and A. Anisimov, Eds., Ch1, pp.1-110, Nova Science: New York (2012).

[25] T. Adunson, B. Gebhart, An experimental and analytical study of natural convection with appreciable thermal radiation effects, J. Fluid Mechanics, Vol. 52, 1972, pp. 57-95.

[26] R. Cortell, A numerical tackling on Sakiadis flow with thermal radiation. Chin Physics Let: 25, 2008, pp. 1340-1342.

[27] J. N. Reddy, An Introduction to the Finite Element Method, McGraw-Hill, New York (1985).

[28] S. R. Rao, The Finite Element Method in Engineering, 2nd Edition, BPCC Wheaton's Ltd., Exeter, USA (1989).

[29] K. J. Bathe, Finite element procedures, Prentice-Hall, New Jersey, USA (1966).

\section{Biography}

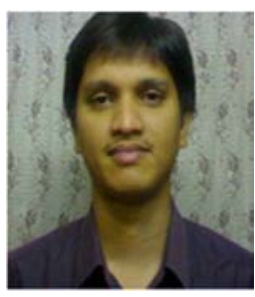

Shamshuddin MD. is working as an Assistant Professor of Mathematics in the Department of Basic Sciences and Humanities, Vaagdevi College of Engineering (Autonomous), Warangal, India. He did his MSc. Mathematics from Osmania university, Hyderabad, in 2002, and obtained M. Phil, from SV university, Tirupati in 2010. He has 14 years of teaching experience. His major field of study is Computational Fluid Dynamics, MHD non-Newtonian flow, Heat and mass transfer flow through porous media, and Boundary value problems. He published more than five national and international journals in Mathematics and Mathematical Physics. 